\title{
Novel Treatment Strategies for Malignant Gliomas Using Neural Stem Cells
}

\author{
Michael C. Oh* and Daniel A. Lim*广 \\ *Department of Neurological Surgery, University of California, San Francisco, San Francisco, California 94143-0112, and \\ ${ }^{\dagger}$ Neurosurgical Section, Surgical Service, San Francisco Veterans Affairs Medical Center, Department of Veterans Affairs, San \\ Francisco, California 94121
}

\begin{abstract}
Summary: Recent studies in stem cell biology have refined our understanding of the origin and progression of cancer. Identification and characterization of endogenous neural stem cells (NSCs), especially those in the adult human brain, have inspired new ideas for selectively targeting and destroying malignant gliomas. Gliomas consist of a heterogeneous population of cells, and some of these cells have characteristics of cancer stem cells. These brain tumor stem cells (BTSCs) share certain characteristics with normal NSCs. It is still unclear, however, whether malignant gliomas in human patients originate from these aberrant BTSCs. Nonetheless, the cellular and molecular
\end{abstract}

similarities between BTSCs and normal NSCs suggest a common research landscape underlying both normal and cancer stem cell biology, wherein findings of one field are relevant to the other. Furthermore, the natural tropism of NSCs to gliomas has generated the idea that modified NSCs can deliver modified genes to selectively destroy malignant brain tumor cells, and even BTSCs, while leaving healthy surrounding neurons intact. These studies and others on the basic biology of both BTSCs and NSCs will be crucial to expanding our treatment strategies for malignant gliomas. Key Words: Neural stem cells, brain tumor stem cells, glioma, gene therapy.

\section{INTRODUCTION}

Identification and characterization of endogenous neural stem cells (NSCs), especially those in the adult human brain, have inspired new ideas about the cellular origin of malignant brain tumors, ${ }^{1-4}$ as well as novel approaches for selectively targeting and destroying malignant gliomas. Malignant gliomas remain one of the most devastating cancers, despite recent advancements in surgical resection and adjuvant therapies. ${ }^{5,6}$ For glioblastoma multiforme, the most common and malignant subtype of glioma, the median survival time remains approximately 14 months. $^{7}$ The resistance of gliomas to current therapeutic measures is in part due to their highly infiltrative nature. Given the invasiveness of glioma cells, with tumorigenic cells often spreading widely into relatively normal brain parenchyma, ${ }^{8,9}$ total surgical resec-

Address correspondence and reprint requests to: Michael C. Oh, M.D., Ph.D., Department of Neurological Surgery, University of California, San Francisco, 505 Parnassus Avenue, San Francisco, CA 94143-0112. E-mail: ohmc@ neurosurg.ucsf.edu. Daniel A. Lim, M.D., Ph.D., Neurosurgical Section, Surgical Service, San Francisco Veterans Affairs Medical Center, Department of Veterans Affairs, 4150 Clement Street, San Francisco, CA 94121. tion is essentially impossible. Furthermore, adjuvant treatment strategies (e.g., external beam radiation and chemotherapy) have not been able to completely treat disease remaining after surgical resection because these treatment modalities cannot specifically target the widely disseminated tumor cells. The toxicity of adjuvant treatments can also result in both systemic illness and cognitive impairment. ${ }^{10}$ New approaches are therefore critical to advancing our ability to treat this devastating disease.

The identification of neural stem cells (NSCs) in the adult human brain and their characterization at the cellular and molecular levels have led to new strategies using such cells to selectively target and destroy malignant gliomas. NSCs can be defined as a population of cells that have the ability to self-renew and produce differentiated neural cell types.

In the adult brain, NSCs have immunocytochemical and ultrastructural characteristics of astrocytes, ${ }^{11}$ and there is a large pool of NSCs found in a region of the brain called the subventricular zone (SVZ), a layer of cells found under the ventricle ependymal cell layer. In rodents, SVZ NSCs give rise to a large number of interneurons for the olfactory bulb and also can generate 
oligodendrocyte and astrocytes. ${ }^{12}$ In the human brain, SVZ NSCs have been also reported to produce neurons for the olfactory bulb, ${ }^{13}$ but this finding remains controversial. ${ }^{14}$ In vivo NSCs, however, are likely quite different from NSCs propagated in vitro for therapeutic purposes. For example, culture conditions that include high concentrations of growth factors can alter differentiation characteristics of NSCs. ${ }^{11,15,16}$ Nonetheless, cultured NSCs derived from endogenous NSCs as well as transitamplifying populations that have dedifferentiated ${ }^{17}$ still satisfy the defining characteristics of NSCs, including self-renewal and production of differentiated neural progeny, and are likely to be useful for novel glioma treatment strategies and cell replacement therapies.

\section{MOLECULAR AND CELLULAR SIMILARITIES BETWEEN NSCS AND BTSCS}

The cellular origin of malignant gliomas is still poorly understood. Some recent studies suggest that gliomas originate from aberrant NSCs, ${ }^{18-23}$ but it is also possible to generate malignant gliomas from dedifferentiated astrocytes. ${ }^{24,25}$ Both NSCs and glioma cells have the ability to self-renew, express cell surface antigen prominin-1 $(\mathrm{CD} 133)^{21,26-28}$ and the NSC marker nestin, and produce both neurons and glia. Furthermore, both cell types appear to be attracted to the same molecular niche, comprised of molecular signals released by either the glioma cells themselves or by surrounding supportive cells. Thus, deciphering the niche and molecular properties of NSCs in the adult brain (reviewed elsewhere; see references $^{29}$ and $^{30}$ ) may lead to novel approaches of treatment that are more selective and potent than current methods.

The adult human brain harbors NSCs in the subventricular zone (SVZ) of the brain ventricles ${ }^{31,32}$ and the subgranular zone (SGZ) of the hippocampal dentate gyrus. ${ }^{33}$ Human brain specimens collected during neurosurgical procedures demonstrate the presence of NSCs in the form of ribbons that line the SVZ of the lateral ventricles. ${ }^{32,34}$ Similar to NSCs found in the rodent brain, ${ }^{31,35}$ these human SVZ NSCs correspond to a subpopulation of glial fibrillary acidic protein (GFAP) expressing astrocytes and are capable of differentiating into neurons, oligodendrocytes, and neurons in vitro. ${ }^{32}$ In the rodent, SVZ NSCs produce a large number of young neuroblasts that migrate to the olfactory bulb, where they differentiate into several different types of interneurons. ${ }^{36}$ Curtis et al. ${ }^{13}$ have proposed that the human SVZ NSCs similarly produce olfactory bulb neurons throughout life; however, this finding has not been observed by others. ${ }^{14}$ Future studies of the human SVZ should shed more light on the fate of normal human SVZ NSCs.

Normal NSCs share molecular and cellular characteristics with BTSCs found in gliomas, and these parallels may provide important therapeutic targets and novel strategies for brain tumor treatment. For instance, it has been shown in studies of SVZ NSCs that the bone morphogenetic protein (BMP) family of signaling molecules causes NSCs to exit the cell cycle and terminally differentiate into astrocytes at the expense of generating other lineages. ${ }^{37,38}$ Given the parallels between NSCs and glioma BTSCs, it was reasonable to believe that BMP signaling would have a similar effect on the malignant phenotype of gliomas in vivo. Intriguingly, Vescovi's group demonstrated that treatment of glioma BTSCs with BMP dramatically reduces the oncogenic potential of specific types of cancer stem cells in a mouse transplantation model. ${ }^{39}$ Clinical trials evaluating BMP in the treatment of brain tumors are currently being planned.

Despite the many similarities between BTSCs and NSCs, there are important differences that are likely to influence treatment strategies. First, unlike normal NSCs, BTSCs likely harbor the oncogenic mutations found in the tumor mass (e.g., activation of oncogenes and loss of tumor suppressor genes). These genetic alterations at the level of the DNA sequence undoubtedly underlie differences between BTSCs and NSCs. Second, there are epigenetic differences in BTSCs that account for variability in the biological behavior of specific subsets of BTSCs in comparison to other BTSCs or normal NSCs. For instance, certain subsets of BTSCs cannot differentiate in response to BMP, and this difference is due to epigenetic silencing (by DNA methylation and the polycomb factor EZH2) of the BMP receptor promoter. ${ }^{40}$ Thus, defining such differences between NSCs and specific BTSCs subtypes will be very important to the design and implementation of glioma treatments that draw upon parallels between NSCs and BTSCs.

\section{NSCS ARE ATTRACTED TO GLIOMAS}

Neural stem cells are known to have high migratory capacity and can travel great distances throughout the brain. This highly migratory property is reminiscent of the invasive quality of malignant gliomas. Although a large conglomeration of tumor cells may be found by radiographic imaging in one location, small numbers of tumor cells can form microsatellites that may be located far away from the main tumor, sometimes even in the contralateral hemisphere. ${ }^{8,41,42}$ The presence of these microsatellite tumors makes complete surgical resection very difficult and is thought to contribute to high recurrence of glioblastomas.

Neural stem cells have extensive tropism to gliomas. ${ }^{43-45}$ Endogenous NSCs are naturally attracted to gliomas $^{44}$ and have the ability to migrate into the tumor mass, even contributing to the tumor bulk. When NSCs are coinjected into rodent brain with glioma cells, NSCs rapidly migrate great distances to track and surround glioma tumors. NSCs injected into the side contralateral 
to malignant tumors have been found to cross the midline and migrate to the tumor. NSCs injected into systemic circulation can also migrate and target intracranial gliomas. Furthermore, glioblastoma cells attract endogenous precursor cells from the SVZ. ${ }^{44}$ These findings could be of high clinical value if translated into human glioma therapy (FIG. 1). NSCs may be used to deliver drugs and/or genes targeted to destroy microsatellite tumor cells that could not be detected intraoperatively or by radiographic imaging and thus escaped surgical resection.

Although there is ample evidence of NSC tropism to gliomas in rodent tumor models, it remains to be verified whether NSCs can track and target glioma cells in the human brain. Furthermore, it will be important to identify the signals involved in attracting NSCs to the gliomas. Once these signaling pathways are better under- stood, one can apply this molecular understanding to track down gliomas, not only for the purpose of targeting therapeutic agents to the tumor, but also to identify and locate residual tumors for further therapy. It will also be important to elucidate the mechanisms involved in regulating the cellular mechanisms of NSC and BTSC migration, as this understanding may be used to identify drugs capable of inhibiting the migratory capacity of BTSCs, thus decreasing their malignant invasiveness. For example, cell surface chemokine receptors, such as $\mathrm{CXCR}^{46}$ and CXCR $4,{ }^{47}$ are potent mediators involved in attracting NSCs to gliomas. NSCs express high concentrations of CXCR4 and blocking these surface receptors block NSC migration towards gliomas. ${ }^{47}$ Furthermore, numerous other cytokines have been implicated in NSC tropism to gliomas, including human growth hormone $(\mathrm{HGH})$, stem cell factor (SCF), urokinase plasmin-

\section{BTSCs tor cells}

\section{Genetically modified NSCs}

A

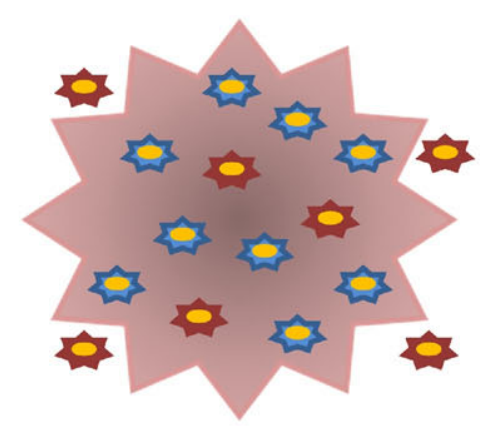

Glioma growth

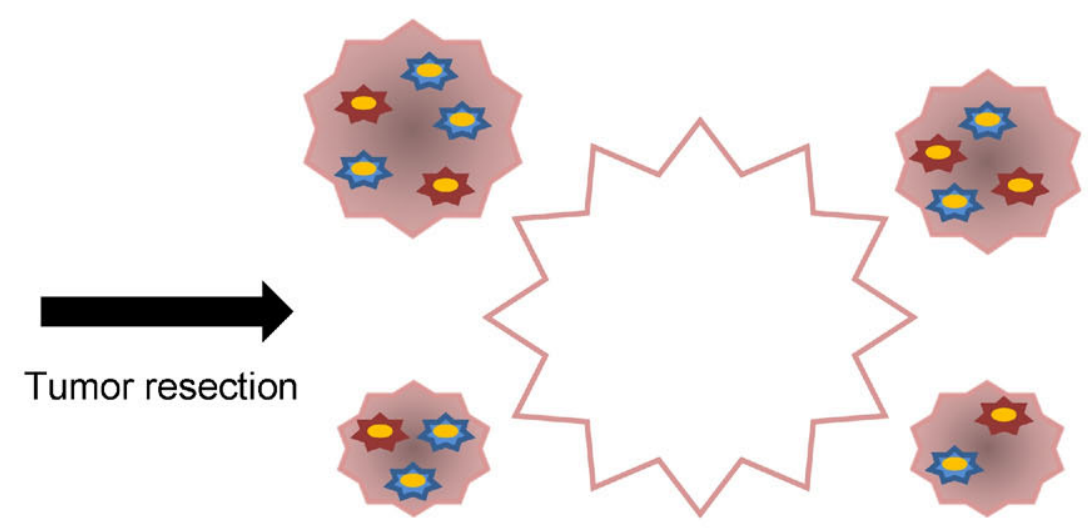

Glioma recurrence

B

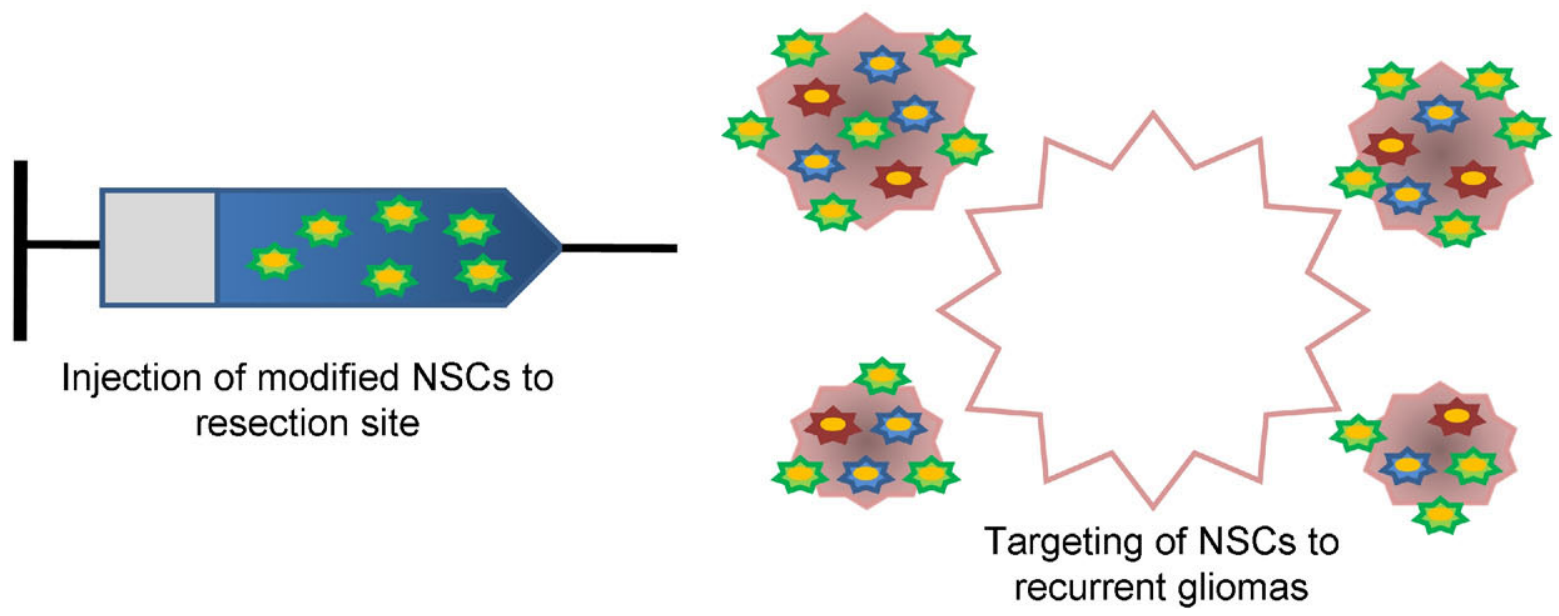

FIG. 1. Glioma proliferation may be supported by brain tumor stem cells (BTSCs). A: Although BTSCs usually comprise a small portion of total tumor bulk, tumor growth and progression (and, more importantly, recurrence) may be dependent on BTSCs. These cells have a high migratory capacity and may form microsatellites of tumor cells far from the primary tumor. Despite attempts to completely resect the primary tumor, disseminated microsatellites of BTSCs may form recurrent tumors. B: Neural stem cells (NSCs) are intrinsically attracted to gliomas and can be used to deliver therapeutic gene products selectively to gliomas. This property could be most useful when modified NSCs carrying genes toxic to tumor cells are injected into the tumor resection cavity to track and kill residual tumor cells. 
ogen activator (uPA), and vascular endothelial growth factor (VEGF). ${ }^{48-51}$

Although it is unclear why NSCs are attracted to gliomas, one possible reason for the recruitment of cells is to repair damaged tissues surrounding the tumor. Demonstrating that NSCs can replace damaged cells and understanding the molecular regulation of this process may lead also to enhanced neurological recovery.

\section{NOVEL TREATMENT STRATEGIES USING NSCS}

Because of the attraction of NSCs to gliomas, NSCs can be used as cellular vehicles for the delivery of therapeutic agents. Recent studies have shown that NSCs can be engineered to deliver a variety of genes that use different mechanisms to selectively destroy gliomas. ${ }^{52}$ These genes, for example, may encode for enzymes that convert prodrugs to toxic metabolites that kill gliomas, or may produce proteins that induce apoptosis or enhance immunity against tumor cells (FIG. 2). Coinjection of unmodified NSCs with glioblastoma cells into the brains of mice results in increased survival and decreased tumor progression, suggesting that NSCs have intrinsic antitumorigenic properties and may release factors that inhibit glioblastoma proliferation. ${ }^{44,45}$ It is critical to further understand the molecular nature of this NSC-mediated antitumor effect for the identification of new therapies for glioblastomas.

One potential mechanism for gene therapy using NSCs is what is called suicide gene therapy. ${ }^{53}$ This is also known as the enzyme-prodrug system, wherein NSCs can be used to deliver genes for an enzyme that can convert a prodrug, administered systemically, into toxic metabolites. These toxic metabolites then selectively target the actively dividing glioma cells while leaving nondividing neurons intact (FIG. 2A).

Two such systems have been tested using NSCs. One system uses cytosine deaminase (CD), which converts nontoxic 5-fluorocytosine (5-FC) into highly toxic 5-fluorouracil (5-FU). This system has been tested for malignant gliomas using an immortalized neural progenitor cell line ST14A that expresses CD. ${ }^{54}$ Although 5-FC has no effect on tumor mass by itself, coinjection of $\mathrm{CD}$ expressing ST14A cells with glioma cells followed by systemic treatment with 5-FC results in approximately a $50 \%$ reduction in tumor mass. Another technique uses the HSV-thymidine kinase-ganciclovir system, wherein HSV-thymidine kinase (HSV-tk) — but not the mammalian thymidine kinase found in the patient-phosphorylates systemically administered ganciclovir to form nucleotide-like precursors that terminate DNA replications in dividing neoplastic cells. Because a retroviral vector is used to transduce the HSV-tk gene, gene transfer occurs only in actively dividing tumor cells, leaving healthy nondividing brain cells intact.

The HSV-tk/ganciclovir system was tested in a phase III clinical trial, using vector-producing cells derived from murine fibroblasts that produced replication-incompetent retrovirus vectors. ${ }^{55}$ However, injecting these vector-producing cells into surgical resection cavities had no significant effects on progression-free survival or median overall survival. This lack of effect was thought to be due to low delivery of HSV-tk gene to the tumor cells. More importantly, the vector-producing cells used in this trial were murine fibroblasts; these cells do not migrate toward glioma cells, which severely limits the delivery of
A. Suicide gene therapy

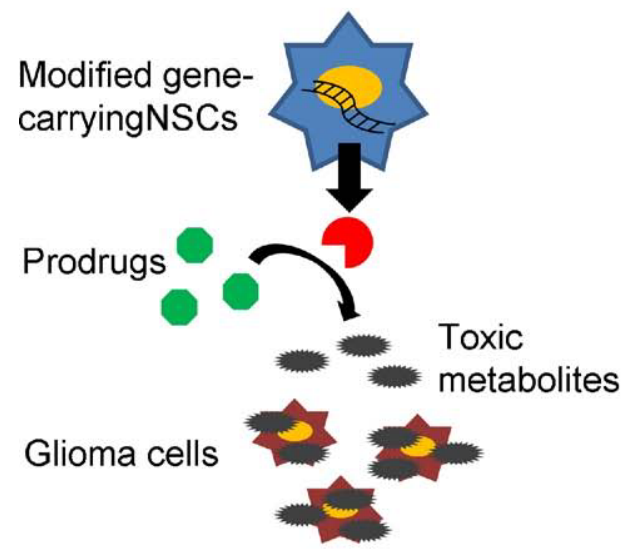

B. Immunomodulators

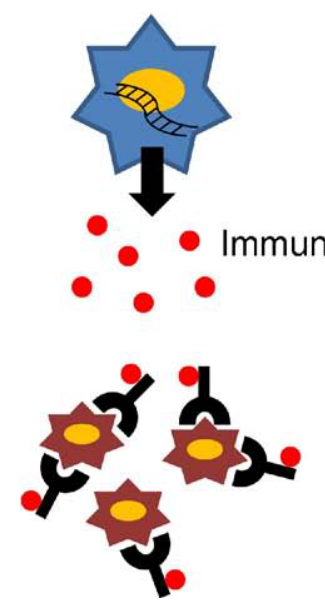

C. Proapoptotic therapy
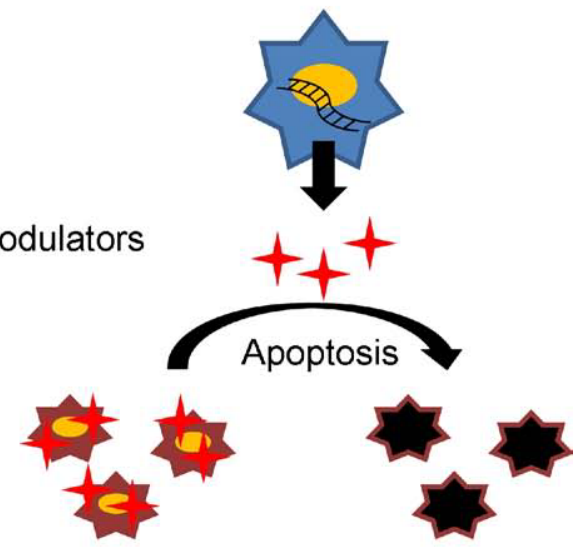

FIG. 2. Neural stem cells (NSCs) are genetically modified to selectively target glioma cells. Modified NSCs have been designed to carry various genes that encode protein products toxic to gliomas. In suicide gene therapy (A), modified NSCs carry genes for an enzyme that converts systemically delivered prodrug into a toxic metabolite that selectively targets glioma cells. Other approaches have used genes that (B) induce specific immunity against gliomas or (C) induce apoptotic pathways selectively in dividing glioma cells to promote glioma cell death. 
HSV-tk to tumor cells. In more recent studies, therefore, NSCs that have intrinsic tropism to gliomas have been used to carry HSV-tk gene. ${ }^{56-58}$ Coinjection of these cells with malignant glioma cells followed by systemic ganciclovir treatment results in prolonged survival and significant inhibition of tumor growth. ${ }^{56}$

This newer system may be more effective in inhibiting tumor progression and growth than the earlier suicide gene systems; however, such a NSC-based treatment strategy has yet to be tested in a clinical trial. Notably, both the CD-5-FC and the HSV-tk-ganciclovir systems show a bystander effect, wherein tumor cells not carrying the suicide genes are also killed, which amplifies the antitumor activity in the suicide gene therapy systems. Although the exact mechanism for the bystander effect is unclear, phosphorylated ganciclovir is thought to be able to pass through gap junctions and kill nontransduced tumor cells nearby. The 5-FU released by cytosine deaminase-expressing cells is also capable of diffusing and killing local tumor cells. ${ }^{59}$ This is an important component to the suicide gene therapy, given that the number of modified NSCs injected into the surgical resection cavity is likely to be small, relative to the residual tumor cells left behind. Thus, the antitumor activity of NSCs carrying the suicide gene is amplified by two different mechanisms. The tropism of NSCs to tumor microsatellites (which may be great distances away from the tumor resection cavity) allows selective homing of suicide genes to targeted areas, and the potent bystander effect can kill nontransduced tumor cells that are near the tumor cells carrying the suicide gene.

NSCs can be also used to deliver target proteins that induce apoptosis of glioma cells (FIG. 2C), such as tumor necrosis factor-related apoptosis-inducing ligand (TRAIL). This is a member of the TNF protein family that has been shown to induce apoptosis in neoplastic cells. ${ }^{60}$ Transplantations of NSCs infected with adenovirus-expressing TRAIL induce apoptosis in established intracranial gliomas, leading to decreases in tumor size. ${ }^{61}$ Furthermore, apoptosis of neoplastic cells is found in pockets of tumors distant from the main tumor, again suggesting that NSCs can track to microsatellites of glioma cells. Thus, using NSCs may allow more direct and selective secretion of this proapoptotic protein near distant microsatellites of gliomas that may be missed during a surgical resection. Another potential target protein is PEX, which is a fragment of metalloproteinase-2 that inhibits glioma angiogenesis. Injection of NSCs transfected with the PEX gene significantly inhibits proliferation and growth of gliomas by inhibiting angiogenesis in the tumor. ${ }^{62}$ Although none of these target proteins have been used in combination, it would be useful to learn whether NSCs that can secrete more than one target protein have additive effects of inhibiting tumor growth.

Immunomodulators have also been shown to be effec- tive in controlling tumor progression (FIG. 2B), and recent studies indicate that interleukin-4 (IL-4), ${ }^{45}$ interleukin-12 (IL-12), ${ }^{63,64}$ and interleukin-23 (IL-23) ${ }^{65}$ are such candidates. Injecting glioblastoma cells (GL261) into either mouse or rat brain results in rapid growth and expansion of tumors, followed by death. Coinjection of IL-4 secreting NSCs prevents tumor growth and progression and significantly lengthens survival. ${ }^{45}$ In fact, coinjection of NSCs not expressing IL-4 also lengthens survival, although less so than IL-4-secreting NSCs, again suggesting that NSCs have intrinsic properties to prevent tumor progression. Thus, the intrinsic capacity to inhibit tumor growth, tropism to gliomas, and capacity to carry genes that are toxic to dividing neoplastic cells are all important characteristics of NSCs that can be applied for development of more effective glioma therapies.

\section{CONCLUSION}

Despite the recent advancements in surgical resections of malignant gliomas and adjuvant therapies, there has been minimal improvement in overall survival. Identification and characterization of NSCs in the adult brain has inspired new approaches that use modified NSCs to target and destroy malignant gliomas. A few important molecular strategies have been discussed here. Future studies of both NSCs and glioma BTSCs will likely lead to identification of new therapeutic targets and strategies, and we envision a synergy of research between these two stem cell types.

The ideal NSC-based therapy for adjuvant therapy of malignant gliomas will have certain properties.

First, the molecular product of the engineered NSC should be a strong inhibitor of tumor growth at low concentrations, because the local concentration of this antitumor activity may be low, due to limited numbers of transplanted NSCs and/or inefficient production of the inhibitors from the NSC itself. Amplification of antitumor effects, such as that provided by the bystander effect, would therefore greatly aid in the success of this treatment strategy.

Second, the NSC-based therapy should be selective for proliferating neoplastic cells, sparing normal neurons and glial cells from harmful side-effects.

Third, the natural tropism of NSCs for gliomas should be maintained or perhaps even enhanced. There may be many tumor satellites located great distances from the resection cavity, away from the implantation site of the engineered NSCs. Furthermore, the number of grafted NSCs will likely be small compared to the patient's tumor burden even after a resection. Thus, efficient NSC homing activity will likely be a key feature of a successful cell-based therapy for malignant gliomas.

Finally, we must exercise caution when using NSCs for human therapy, because there is always the potential 
for tumor growth from grafts of undifferentiated NSC populations. ${ }^{66}$ It may be prudent to have some method of eliminating the transplanted cells (or causing their terminal differentiation) after they have served their function.

If all these criteria are met, then the simple implantation of engineered NSCs to the tumor cavity walls after surgical resection of the primary tumor might be sufficient to allow rapid migration of these NSCs toward the residual tumor microsatellites. Thus, even residual disease located in eloquent areas (e.g., language and motor centers) might be eliminated, while sparing the normal neurons and glia from the collateral damage of direct surgical manipulation. Clearly, continued studies of malignant gliomas, BTSCs, and the molecular and cellular characteristics of NSCs will be vital for the development of new adjuvant therapies for malignant gliomas.

Acknowledgments: We thank the reviewers for their helpful comments and Benjamin Sherman for his editorial assistance and intellectual input.

\section{REFERENCES}

1. Berger F, Gay E, Pelletier L, Tropel P, Wion D. Development of gliomas: potential role of asymmetrical cell division of neural stem cells. Lancet Oncol 2004;5:511-514.

2. Holland EC. Progenitor cells and glioma formation. Curr Opin Neurol 2001;14:683-688.

3. Oliver TG, Wechsler-Reya RJ. Getting at the root and stem of brain tumors. Neuron 2004;42:885-888.

4. Sanai N, Alvarez-Buylla A, Berger MS. Neural stem cells and the origin of gliomas. N Engl J Med 2005;353:811-822.

5. Sathornsumetee S, Rich JN. Designer therapies for glioblastoma multiforme. Ann N Y Acad Sci 2008;1142:108-132.

6. Wen PY, Kesari S. Malignant gliomas in adults. N Engl J Med 2008;359:492-507.

7. Stupp R, Hegi ME, Mason WP, et al; European Organisation for Research and Treatment of Cancer Brain Tumour and Radiation Oncology Groups; National Cancer Institute of Canada Clinical Trials Group. Effects of radiotherapy with concomitant and adjuvant temozolomide versus radiotherapy alone on survival in glioblastoma in a randomised phase III study: 5-year analysis of the EORTC-NCIC trial. Lancet Oncol 2009;10:459-466.

8. Giese A, Bjerkvig R, Berens ME, Westphal M. Cost of migration: invasion of malignant gliomas and implications for treatment. J Clin Oncol 2003;21:1624-1636.

9. Lefranc F, Brotchi J, Kiss R. Possible future issues in the treatment of glioblastomas: special emphasis on cell migration and the resistance of migrating glioblastoma cells to apoptosis. J Clin Oncol 2005;23:2411-2422.

10. DeAngelis LM. Benefits of adjuvant chemotherapy in high-grade gliomas. Semin Oncol 2003;30:15-18.

11. Lim DA, Alvarez-Buylla A. Neural stem cells in the adult brain: implications of their glial characteristics. In: Rao MS, editor. Neural development and stem cells. 2nd ed. Totowa, NJ: Humana Press, 2006:29-47.

12. Ihrie RA, Alvarez-Buylla A. Cells in the astroglial lineage are neural stem cells. Cell Tissue Res 2008;331:179-191.

13. Curtis MA, Kam M, Nannmark U, et al. Human neuroblasts migrate to the olfactory bulb via a lateral ventricular extension. Science 2007;315:1243-1249.

14. Sanai N, Berger MS, Garcia-Verdugo JM, Alvarez-Buylla A. Comment on "Human neuroblasts migrate to the olfactory bulb via a lateral ventricular extension." Science 2007;318:393; author reply 393.
15. Kondo T, Raff M. Oligodendrocyte precursor cells reprogrammed to become multipotential CNS stem cells. Science 2000;289:17541757.

16. Suhonen JO, Peterson DA, Ray J, Gage FH. Differentiation of adult hippocampus-derived progenitors into olfactory neurons in vivo. Nature 1996;383:624-627.

17. Doetsch F, Petreanu L, Caille I, Garcia-Verdugo JM, AlvarezBuylla A. EGF converts transit-amplifying neurogenic precursors in the adult brain into multipotent stem cells. Neuron 2002;36: $1021-1034$.

18. Galli R, Binda E, Orfanelli U, et al. Isolation and characterization of tumorigenic, stem-like neural precursors from human glioblastoma. Cancer Res 2004;64:7011-7021.

19. Hemmati HD, Nakano I, Lazareff JA, et al. Cancerous stem cells can arise from pediatric brain tumors. Proc Natl Acad Sci U S A 2003;100:15178-15183.

20. Piccirillo SG, Combi R, Cajola L, et al. Distinct pools of cancer stem-like cells coexist within human glioblastomas and display different tumorigenicity and independent genomic evolution. Oncogene 2009;28:1807-1811.

21. Singh SK, Hawkins C, Clarke ID, et al. Identification of human brain tumour initiating cells. Nature 2004;432:396-401.

22. Yi L, Zhou ZH, Ping YF, et al. Isolation and characterization of stem cell-like precursor cells from primary human anaplastic oligoastrocytoma. Mod Pathol 2007;20:1061-1068.

23. Alcantara Llaguno S, Chen J, Kwon $\mathrm{CH}$, et al. Malignant astrocytomas originate from neural stem/progenitor cells in a somatic tumor suppressor mouse model. Cancer Cell 2009;15:45-56.

24. Bachoo RM, Maher EA, Ligon KL, et al. Epidermal growth factor receptor and Ink4a/Arf: convergent mechanisms governing terminal differentiation and transformation along the neural stem cell to astrocyte axis. Cancer Cell 2002;1:269-277.

25. Dai C, Celestino JC, Okada Y, et al. PDGF autocrine stimulation dedifferentiates cultured astrocytes and induces oligodendrogliomas and oligoastrocytomas from neural progenitors and astrocytes in vivo. Genes Dev 2001;15:1913-1925.

26. Singh SK, Clarke ID, Hide T, Dirks PB. Cancer stem cells in nervous system tumors. Oncogene 2004;23:7267-7273.

27. Singh SK, Clarke ID, Terasaki M, et al. Identification of a cancer stem cell in human brain tumors. Cancer Res 2003;63:5821-5828.

28. Uchida N, Buck DW, He D, et al. Direct isolation of human central nervous system stem cells. Proc Natl Acad Sci U S A 2000;97: $14720-14725$.

29. Alvarez-Buylla A, Lim DA. For the long run: maintaining germinal niches in the adult brain. Neuron 2004;41:683-686.

30. Lim DA, Huang YC, Alvarez-Buylla A. The adult neural stem cell niche: lessons for future neural cell replacement strategies. Neurosurg Clin N Am 2007;18:81-92, ix.

31. Doetsch F, Caille I, Lim DA, Garcia-Verdugo JM, Alvarez-Buylla A. Subventricular zone astrocytes are neural stem cells in the adult mammalian brain. Cell 1999;97:703-716.

32. Sanai N, Tramontin AD, Quinones-Hinojosa A, et al. Unique astrocyte ribbon in adult human brain contains neural stem cells but lacks chain migration. Nature 2004;427:740-744.

33. Eriksson PS, Perfilieva E, Björk-Eriksson T, et al. Neurogenesis in the adult human hippocampus. Nat Med 1998;4:1313-1317.

34. Quiñones-Hinojosa A, Sanai N, Soriano-Navarro M, et al. Cellular composition and cytoarchitecture of the adult human subventricular zone: a niche of neural stem cells. J Comp Neurol 2006;494: 415-434.

35. Garcia AD, Doan NB, Imura T, Bush TG, Sofroniew MV. GFAPexpressing progenitors are the principal source of constitutive neurogenesis in adult mouse forebrain. Nat Neurosci 2004;7:12331241.

36. Alvarez-Buylla A, Kohwi M, Nguyen TM, Merkle FT. The heterogeneity of adult neural stem cells and the emerging complexity of their niche. Cold Spring Harb Symp Quant Biol 2008;73:357-365.

37. Gross RE, Mehler MF, Mabie PC, et al. Bone morphogenetic proteins promote astroglial lineage commitment by mammalian subventricular zone progenitor cells. Neuron 1996;17:595-606.

38. Lim DA, Tramontin AD, Trevejo JM, et al. Noggin antagonizes BMP signaling to create a niche for adult neurogenesis. Neuron 2000;28:713-726. 
39. Piccirillo SG, Reynolds BA, Zanetti N, et al. Bone morphogenetic proteins inhibit the tumorigenic potential of human brain tumourinitiating cells. Nature 2006;444:761-765.

40. Lee J, Son MJ, Woolard K, et al. Epigenetic-mediated dysfunction of the bone morphogenetic protein pathway inhibits differentiation of glioblastoma-initiating cells. Cancer Cell 2008;13:69-80.

41. Matsukado Y, MacCarty CS, Kernohan JW. The growth of glioblastoma multiforme (astrocytomas, grades 3 and 4) in neurosurgical practice. J Neurosurg 1961;18:636-644.

42. Demuth T, Berens ME. Molecular mechanisms of glioma cell migration and invasion. J Neurooncol 2004;70:217-228.

43. Aboody KS, Brown A, Rainov NG, et al. Neural stem cells display extensive tropism for pathology in adult brain: evidence from intracranial gliomas. Proc Natl Acad Sci U S A 2000;97:1284612851.

44. Glass R, Synowitz M, Kronenberg G, et al. Glioblastoma-induced attraction of endogenous neural precursor cells is associated with improved survival. J Neurosci 2005;25:2637-2646.

45. Benedetti S, Pirola B, Pollo B, et al. Gene therapy of experimental brain tumors using neural progenitor cells. Nat Med 2000;6:447450.

46. Honeth G, Staflin K, Kalliomaki S, Lindvall M, Kjellman C. Chemokine-directed migration of tumor-inhibitory neural progenitor cells towards an intracranially growing glioma. Exp Cell Res 2006;312:1265-1276.

47. Ehtesham M, Yuan X, Kabos P, et al. Glioma tropic neural stem cells consist of astrocytic precursors and their migratory capacity is mediated by CXCR4. Neoplasia 2004;6:287-293.

48. Gutova M, Najbauer J, Frank RT, et al. Urokinase plasminogen activator and urokinase plasminogen activator receptor mediate human stem cell tropism to malignant solid tumors. Stem cells 2008;26:1406-1413.

49. Kendall SE, Najbauer J, Johnston HF, et al. Neural stem cell targeting of glioma is dependent on phosphoinositide 3-kinase signaling. Stem cells 2008;26:1575-1586.

50. Najbauer J, Danks MK, Schmidt NO, Kim SU, Aboody KS. Neural stem cell-mediated therapy of primary and metastatic solid tumors. In: Bertolotti R, Ozawa K, editors. Progress in gene therapy, Autologous and cancer stem cell gene therapy. Progress in Gene Therapy. Vol. 3. Hackensack, NJ: World Scientific Publishing Co., 2007:335-372.

51. Zhao D, Najbauer J, Garcia E, et al. Neural stem cell tropism to glioma: critical role of tumor hypoxia. Mol Cancer Res 2008;6: $1819-1829$.

52. Aboody KS, Najbauer J, Danks MK. Stem and progenitor cellmediated tumor selective gene therapy. Gene Ther 2008;15:739752.
53. Aghi M, Hochberg F, Breakefield XO. Prodrug activation enzymes in cancer gene therapy. J Gene Med 2000;2:148-164.

54. Barresi V, Belluardo N, Sipione S, et al. Transplantation of prodrug-converting neural progenitor cells for brain tumor therapy. Cancer Gene Ther 2003;10:396-402.

55. Rainov NG. A phase III clinical evaluation of herpes simplex virus type 1 thymidine kinase and ganciclovir gene therapy as an adjuvant to surgical resection and radiation in adults with previously untreated glioblastoma multiforme. Hum Gene Ther 2000;11:2389-2401.

56. Li S, Tokuyama T, Yamamoto J, et al. Bystander effect-mediated gene therapy of gliomas using genetically engineered neural stem cells. Cancer Gene Ther 2005;12:600-607.

57. Li S, Tokuyama T, Yamamoto J, et al. Potent bystander effect in suicide gene therapy using neural stem cells transduced with herpes simplex virus thymidine kinase gene. Oncology 2005;69:503508.

58. Herrlinger U, Woiciechowski C, Sena-Esteves M, et al. Neural precursor cells for delivery of replication-conditional HSV-1 vectors to intracerebral gliomas. Mol Ther 2000;1:347-357.

59. Huber BE, Austin EA, Richards CA, Davis ST, Good SS. Metabolism of 5-fluorocytosine to 5-fluorouracil in human colorectal tumor cells transduced with the cytosine deaminase gene: significant antitumor effects when only a small percentage of tumor cells express cytosine deaminase. Proc Natl Acad Sci U S A 1994;91: $8302-8306$.

60. Walczak H, Miller RE, Ariail K, et al. Tumoricidal activity of tumor necrosis factor-related apoptosis-inducing ligand in vivo. Nat Med 1999;5:157-163.

61. Ehtesham M, Kabos P, Gutierrez MA, et al. Induction of glioblastoma apoptosis using neural stem cell-mediated delivery of tumor necrosis factor-related apoptosis-inducing ligand. Cancer Res 2002;62:7170-7174.

62. Kim SK, Cargioli TG, Machluf M, et al. PEX-producing human neural stem cells inhibit tumor growth in a mouse glioma model. Clin Cancer Res 2005;11:5965-5970.

63. Ehtesham M, Kabos P, Kabosova A, et al. The use of interleukin 12 -secreting neural stem cells for the treatment of intracranial glioma. Cancer Res 2002;62:5657-5663.

64. Yang SY, Liu H, Zhang JN. Gene therapy of rat malignant gliomas using neural stem cells expressing IL-12. DNA Cell Biol 2004;23: 381-389.

65. Yuan X, Hu J, Belladonna ML, Black KL, Yu JS. Interleukin-23expressing bone marrow-derived neural stem-like cells exhibit antitumor activity against intracranial glioma. Cancer Res 2006;66: $2630-2638$.

66. Amariglio N, Hirshberg A, Scheithauer BW, et al. Donor-derived brain tumor following neural stem cell transplantation in an ataxia telangiectasia patient. PLoS Med 2009;6(2):e1000029. 